\title{
Coordinated Grid and Place Cell Replay during Rest
}

H. Freyja Ólafsdóttir ${ }^{1^{*}}$, Francis Carpenter ${ }^{1,2}$, \& Caswell Barry ${ }^{{ }^{*}}$.

1. Department of Cell and Developmental Biology, University College London, London.

2. Institute of Neurology, University College London, London.

*to whom correspondence should be addressed: h.olafsdottir@ucl.ac.uk, caswell.barry@ucl.ac.uk.

Key words: grid cell, place cell, hippocampus, entorhinal cortex, replay, memory consolidation, navigation

Hippocampal replay has been hypothesized to underlie memory consolidation and navigational planning, yet the involvement of grid cells in replay is unknown. During replay we found grid cells were spatially coherent with place cells, encoding locations $11 \mathrm{~ms}$ delayed vs the hippocampus - with directionally-modulated grid cells and forward replay exhibiting the greatest coherence with CA1. This suggests grid cells are engaged during the consolidation of spatial memories to the neocortex.

During exploration, the activity of place ${ }^{1}$ and grid cells ${ }^{2}$ represent self-location. Together these cells have been hypothesized to support spatial memory ${ }^{1,3}$ and navigation ${ }^{3,4}$. Hippocampal replay ${ }^{5,6}-$ the reactivation of place cell sequences during immobility and sleep - has been proposed as a mechanism for consolidation ${ }^{5}$ and route planning ${ }^{7}$, yet the involvement of grid cells remains unknown. Potentially, spatially coherent place and grid cell activity may emerge during replay as the hippocampus broadcasts memory traces to the cortex ${ }^{8}$. To study the involvement of grid cells in replay we recorded concurrently from rodent medial entorhinal cortex layers 5/6 (MECV\&VI) and hippocampus (CA1) during trackrunning and subsequent rest. We report robust coherence between place and grid cell spatial representations during hippocampal replay.

A total of 43 grid cells were recorded across 11 sessions from 6 rats. Concurrently, 34-72 place cells were recorded in each session, 592 in total (Fig. 1a-d, Supplementary Fig. 1,2, Supplementary Table 1). During subsequent rest, we identified putative replay events based on place cell activity (Figure 1e, see Online Methods). A Bayesian decoding algorithm ${ }^{9}$ and a trajectory-fitting procedure was used to reconstruct position and score the replay ${ }^{9}$ (Supplementary Fig. 3). Robust replay events exhibiting clear, straight trajectories (each $p<0.2$ vs own shuffle) were used for further analyses. On average, during replay events, grid cell activity was higher than during non-replay periods ${ }^{10}(2.19 \mathrm{~Hz}(S D=1.74)$ vs $\left.1.33 \mathrm{~Hz}(\mathrm{SD}=1.61), t_{(43)}=3.24, \mathrm{p}=0.00023\right)$, with peak grid cell activity lagging that of place cells by $10 \mathrm{~ms}$ (Fig. 1f, Supplementary Fig. 4). To investigate grid-place cell spatial coherence, we assessed the similarity of the grid and place cell representations during replay events in which both were active. 
Specifically, we superimposed the trajectory derived from a hippocampal replay event onto the decoded representation from concurrently recorded grid cell spikes (Supplementary Fig. 5). The position represented by grid cells during replay events was similar to that represented by the place cell trajectories (Fig. 2a, Supplementary Fig. 6), exceeding the coherence obtained by pairing a grid cell event with a random place cell event from the same session $(p<0.0001$ area under the curve (AUC) test, see Online Methods, Fig. 2b, Supplementary Fig. 5); comparisons against shuffled distributions generated by permuting grid cell ratemaps ( $p<0.0001$ AUC) and spike times $(p<0.001$ AUC) corroborated this finding (Supplementary Fig. 7,8). Importantly, grid-place coherence also exceeded chance levels when analyses was limited to just the strongest ( $p<0.025$ vs own shuffle) place cell replay events ( $p<0.0001$ AUC, Supplementary Fig. 9 ) and did not exceed chance levels for the least robust $(p>0.5)$ place cell events ( $p=0.17$ AUC, Supplementary Fig. 10).

To confirm these results, we constructed an 'event-ratemap' for each grid cell using the spikes emitted during replay events and the position decoded from concurrent place cell activity (see Online Methods). Event-ratemaps and ratemaps generated from track running were similar (mean Pearson correlation = 0.10 , SEM $=0.033$, Fig. $2 c, d$ ) and exceeded the correlations obtained from two shuffling procedures (ratemap shuffle $\mathrm{W}_{(78)}=763, \mathrm{p}=0.0079$; grid spike times shuffle, $\mathrm{W}_{(78)}=703, \mathrm{p}=0.01$ ). In summary, we employed two distinct methods to assess spatial coherence between grid and place cells during replay events, confirming that these cell types are closely coordinated during hippocampal replay.

Forward replay - events that preserve place field sequences experienced during wakefulness - have been linked to consolidation and planning ${ }^{5,7}$ and reverse replay to reward learning ${ }^{6}$. Consequently, we assessed coordination between grid and place cells during forward and reverse replay events separately. Grid cells were more likely to be active during forward than reverse events (forward: $639 / 1127(57 \%)$ vs reverse: $\left.315 / 699(45 \%), \chi^{2}=23.41, p<0.0001\right)$ and grid-place cell spatial coherence was greater for forward than reverse replay ( $p=0.0006$ AUC, Fig. 3a). This difference did not result from the unequal number of forward and reverse events - equating the number of events by down-sampling did not eliminate the difference $(p<0.0001)$. Forward and reverse events both exhibited grid-place coherence that significantly exceeded chance levels (Forward: $p<0.0001$ AUC, Reverse: $p=0.0093$ AUC, Figure 3a).

Next, we examined the temporal relationship between grid and place cell spatial coherence during replay events. If grid cell participation in replay results from a consolidation mechanism ${ }^{5}$, the location encoded by grid cells might lag the place cell trajectory - similar to the $10 \mathrm{~ms}$ lag seen in peak rates between the two regions (Fig. 1f). We investigated grid-place cell coherence for time lags between $\pm 60 \mathrm{~ms}$, finding a significant effect of time for forward but not reverse events (forward: $F_{(24,4320)}=9.26$, $p<0.0001$, reverse: $\left.F_{(24,1464)}=0.37, p>0.05\right)$. Moreover, for forward events we found the highest gridplace cell coherence when grid cell spike times were shifted back by an average of $11 \mathrm{~ms}$ relative to place cells (Fig. $3 b$ ) - analysis of the distribution of best time shifts across forward events revealed a unimodal distribution peaked at $8 \mathrm{~ms}$ (Fig. 3c). 
Finally, we investigated the relationship between the strength of replay coherence for individual grid cells (indexed by their mean spatial coherence) and the extent of their modulation by head direction and hexagonally symmetry in the open field (gridness ${ }^{11}$ ). Directional modulation was found to correlate with replay coherence (Spearman's $r=0.61, p=0.000093$ fig. $3 d$ ) such that directional grid cells (KL divergence $>0.15)^{12}$ exhibited higher coherence with place cells than non-directional grid cells $(0.16$ ( $S D=0.082)$ vs 0.082 ( $S D=0.030), t_{(35)}=3.57, p=0.0011, p<0.0001$ AUC test). Yet, both cell types showed significant grid-place cell coherence (directional cells: $p<0.0001$ AUC, non-directional cells: $p<0.0001$ AUC, Supplementary Fig. 11) as well as a lag relative to the CA1 encoded location (directional cells: $F_{(24,1056)}=4.150 p<0.0001$, non-directional cells: $F_{(24,3312)}=3.060, p<0.0001$, Supplementary Fig. 12$)$. Grid-place cell coherence was not modulated by hexagonal symmetry (Spearman's $r=0.14, p=0.41$, Supplementary Fig. 13).

To conclude, we report that hippocampal place cells and MECV\&VI grid cells are closely coordinated during replay, suggesting that replay may be a general property of networks encoding self-location. Moreover, grid cells are more active during forward replay events during which they exhibit greater spatial coherence with place cells. During these events the position encoded by MECV\&VI lags behind CA1, suggesting replay sequences originate in the hippocampus and propagate to the MEC. As the coordination occurs in the absence of sensory cues it plausibly originates from the internal dynamics of the hippocampal network ${ }^{13}$. Together this supports the view that replay during rest is the mechanism by which memories are consolidated to the neocortex ${ }^{8}-M E C V \& V I$ being the primary cortical output target of the hippocampus. It remains to be seen if grid cells in superficial MEC are engaged during ontrack replay or navigational planning ${ }^{4}$.

\section{Acknowledgements}

This work was supported by the Wellcome Trust and the Royal Society (101208/Z/13/Z). The authors thank Neil Burgess, Kate Jeffery, Tom Wills, Daniel Bush, and Aman Saleem for comments on the manuscript.

\section{Author Contributions}

H.F.O. and C.B. conceived of the project jointly. H.F.O. and F.C. performed surgeries. H.F.O. carried out experiments. H.F.O. and C.B. performed the analyses. All authors wrote the manuscript.

\section{Competing Financial Interests Statement}

The authors declare no competing financial interests.

\section{References}

$1 \quad$ O'Keefe, J. \& Nadel, L. (Claredon, Oxford, 1978).

2 Hafting, T., Fyhn, M., Molden, S., Moser, M. B. \& Moser, E. I. Nature 436, 801-806, doi:10.1038/nature03721 (2005).

3 McNaughton, B. L., Battaglia, F. P., Jensen, O., Moser, E. I. \& Moser, M. B. Rev Neurosci 7, 663678, doi:10.1038/nrn1932 (2006). 
Bush, D., Barry, C., Manson, D. \& Burgess, N. Neuron 87, 507-520, doi:10.1016/j.neuron.2015.07.006 (2015).

$5 \quad$ Wilson, M. A. \& McNaughton, B. L. Science 265, 676-679 (1994).

6 Foster, D. J. \& Wilson, M. A. Nature 440, 680-683, doi:10.1038/nature04587 (2006).

$7 \quad$ Pfeiffer, B. E. \& Foster, D. J. Nature 497, 74-79, doi:10.1038/nature12112 (2013).

8 Marr, A. Philos Trans R Soc Lond B Biol Sci 262, 23-81 (1971).

9 Olafsdottir, H. F., Barry, C., Saleem, A. B., Hassabis, D. \& Spiers, H. J. elife 4, e06063, doi:10.7554/eLife.06063 (2015).

10 Chrobak, J. J. \& Buzsaki, G. J Neurosci 14, 6160-6170 (1994).

11 Boccara, C. N. et al. Nat Neurosci 13, 987-994, doi:10.1038/nn.2602 (2010).

12 Doeller, C. F., Barry, C. \& Burgess, N. Nature 463, 657-661, doi:10.1038/nature08704 (2010).

13 Pastalkova, E., Itskov, V., Amarasingham, A. \& Buzsaki, G. Science 321, 1322-1327, doi:10.1126/science.1159775 (2008).

Figure 1: Experimental procedure. (a) Rats ran 20 laps on a Z-shaped track (Track Running), and were then placed in a rest enclosure (Rest) for an hour and a half, and finally completed an open field foraging session (Forage). (b\&c) Replay analyses were based on linearized track ratemaps, single example grid (b) and place (c) cell shown. (d) Open field ratemaps were used to identify grid and directionally modulated cells, example grid cell shown. (e) Example event. Top: raster plot of place cell (red) and grid cell (blue) spikes recorded during rest. Dashed vertical lines mark start and end of replay event (x-axis: time, $y$-axis: arbitrary cell IDs). Bottom: smoothed multi-unit place (red) and grid (blue) activity ( $x$-axis: time, $y$-axis: spike rate $(\mathrm{Hz})$ ). (f) Peri-stimulus time histogram (PSTH) of grid cell activity during replay events, centred on the middle of the event ( $x$-axis, time, $y$-axis, number of spikes).

Figure 2: Grid-place cell spatial coherence during replay. (a) Six example reconstructed replay events based on concomitant place cell (red boxes) and grid cell (blue boxes) spikes (x-axis time, $y$ axis linearized position). White lines mark the extent of the line-fit based on place cell activity. Title indicates animal ID and strength of line-fit. (b) Bootstrapped cumulative distribution of grid-place coherence (place cell line-fit imposed on grid cells) obtained for all animals (blue line), shaded area shows 1SD ( $x$-axis coherence scores, $y$-axis cumulative proportion of events). (Black line) distribution from shuffle (random pairing of grid and place cell events). Inset, difference between data and shuffle; negative difference indicates greater coherence in the data distribution. (c) Cumulative distribution of correlations between track and event ratemaps for grid cells (blue, $x$-axis Person correlation ( $r$ ), $y$-axis proportion of grid cells). (Black line) distribution from shuffle (event and track ratemaps correlated at all lags). (Green line) distribution of correlations between track ratemap and ratemaps derived from position decoded from place cell activity during track running. (d) Three example track (green) and event ratemaps (blue). Grid cell spikes in (a): Top row left: 2 cells, 3 spikes; middle: 3 cells, 4 spikes; right: 3 cells, 3 spikes. Bottom row left: 1 cell, 1 spike; middle: 1 cell, 1 spike; right: 2 cells, 2 spikes.

Figure 3: Grid-place cell coherence is stronger for forward replay and directionally modulated cells. (a) Bootstrapped cumulative distribution of spatial coherence scores for forward (orange), reverse (grey) and both (blue) replay events, shaded area 1SD (x-axis grid-place cell coherence, y-axis cumulative proportion of events). Inset, difference between data and shuffle. (b) Mean grid-place cell 
coherence vs time shift of grid cell spikes for forward (orange) and reverse (grey) events ( $x$-axis, grid cell time shift, negative indicates grid spikes were moved back in time relative to place cell spikes, $y$ axis, mean grid-place coherence, shaded area shows SEM). (c) Distribution of time shift associated with highest grid-place cell coherence (x-axis, grid cell time shift, $y$-axis, number of events). (d) Gridplace cell coherence as a function of grid cell directional modulation ( $\mathrm{x}$-axis, $\mathrm{KL}$ divergence of each grid cell, y-axis, mean spatial coherence with place cells during replay, title, Spearman rank order correlation, open circles: grid cells with $\mathrm{KL}<0.15)$.

\section{Online Methods}

\section{Animals and surgery}

Six male Lister Hooded rats were used in this study. All procedures were approved by the UK Home Office, subject to the restrictions and provisions contained in the Animals (Scientific Procedures) Act of 1986. All rats (330-400g at implantation) received two microdrives, each carrying eight tetrodes of twisted $17 \mu \mathrm{m} \mathrm{HM-L} \mathrm{coated} \mathrm{platinum} \mathrm{iridium} \mathrm{wire}(90 \%$ and $10 \%$, respectively; California Fine Wire), targeted to the right CA1 (ML: $2.2 \mathrm{~mm}$, AP: $3.8 \mathrm{~mm}$ posterior to Bregma) and left medial entorhinal cortex $(\mathrm{MEC})(\mathrm{ML}=4.5 \mathrm{~mm}, \mathrm{AP}=0.3-0.7$ anterior to the transverse sinus, angled between 8-10). Wires were platinum plated to reduce impedance to $200-300 \mathrm{k} \Omega$ at $1 \mathrm{kHz}$. After rats had recovered from surgery they were maintained at $90 \%$ of free-feeding weight with ad libitum access to water, and were housed individually on a 12-hr light/dark cycle.

\section{Recording}

Screening was performed post-surgically after a 1-week recovery period. An Axona recording system (Axona Ltd.) was used to acquire the single-units and positional data (for details of the recording system and basic recording protocol see Barry et $\mathrm{al}^{1}$ ). The position and head direction of the animals was inferred using an overhead video camera to record the location of two light-emitting diode (LED) mounted on the animals' head-stages $(50 \mathrm{~Hz})$. Tetrodes were gradually advanced in 62.5um steps across days until place cells (CA1) or grid cells (MEC) were found.

\section{Experimental apparatus and protocol}

The experiment was run during the animal's light period, to facilitate rest during the rest session. During track running sessions animals shuttled back and forwards on a Z-shaped track comprised of $10 \mathrm{~cm}$ wide runways covered with black paint, raised $75 \mathrm{~cm}$ off the ground. The two parallel sections of the $Z$ $(190 \mathrm{~cm}$ each) were connected by a diagonal section $(220 \mathrm{~cm})$. The entire track was surrounded by plain black curtains. Animals were pre-trained to run on the track, taking between 3 and 6 days before they would shuttle fluently from one end to the other. At the start of each session, rats were placed at one end of the Z-track. The same end was used as a starting location for every day of the experiment and for every rat. The ends and corners of the track were baited with sweetened rice to encourage running from one end to the other. In each session rats completed 20 full laps (30-45min). 
Following the track session, rats were placed in the rest enclosure for an hour and a half. The rest enclosure consisted of a cylindrically shaped environment $(18 \mathrm{~cm}$ diameter, $61 \mathrm{~cm}$ high) with a towel placed at the bottom and was located outside of the curtains which surrounded the Z-track. Animals were not able to see the surrounding room while in the rest enclosure. Prior to recording, rats had been familiarised with the rest environment for at least 7 days.

Following the rest session, rats completed a 20min foraging trial in a familiar open field environment. Recording made during this period provided the basis on which spatially modulated cells were functionally classified.

As we did not use experimental groups, randomisation and experimenter blinding were not applicable.

\section{Data inclusion/exclusion}

Once animals were experienced and ran well on the track (following 3-6days of training) we considered that any session with at least 30 place cells and 1 grid cell met our minimum requirements and hence were eligible for analysis. The number of sessions which fulfilled these criteria varied between animals (1-3). Importantly, if multiple sessions were included from the same animal, we ensured the different sessions did not contain the same grid cells (based on history of electrode movement, waveforms, and spatial ratemaps in the open field screening sessions).

The criteria for including place and grid cells are described below ('Functional classification' and 'Data analysis' sub-sections) as well as the criteria for including replay events ('Data analysis' sub-section). All data that met these criteria were included in all of the analyses.

\section{Functional classification}

Spatially modulated MEC cells were classified as grid cells using a shuffling procedure similar to that applied elsewhere ${ }^{2,3}$. Specifically, the hexagonal regularity of each cell was assessed using two methods, the 'standard' gridness measure ${ }^{4}$ and 'modified' gridness measure ${ }^{3}$. For each method the values calculated for each cell were compared with a null distribution of 100 values obtained by calculating the gridness values of data in which the cell's spike train had been randomly permuted relative to the position of the animal by at least $30 \mathrm{~s}$. A cell was considered to be a grid cell and admitted to the main analysis if its standard or modified gridness value exceeded the $97.5^{\text {th }}$ percentile of the matching null distribution (43 grid cells from 6 rats were identified).

Following Doeller et al. ${ }^{5}$ the extent of directional modulation exhibited by each grid cell was assessed by calculating the Kullback-Leibler (KL) divergence between the cell's polar rate map and a uniform circular distribution with equal mean:

$$
D_{K L}=\sum_{i} \frac{\tau_{1}(i) \log \left(\tau_{1}(i)\right)}{\tau_{2}(i)}
$$


Where $\tau_{1}(i)$ is the value in the ith bin of a polar rate map normalised to have area 1 (as a probability distribution) and $\tau_{2}(i)$ is the ith bin of a uniform probability distribution with the same number of bins as ${ }_{1}$. Grid cells with $\mathrm{KL}$ divergence greater than 0.15 were considered to be directional ${ }^{5,6}$ (13 of 43 cells).

\section{Data analysis}

Ratemaps for runs on the Z-track were generated after first excluding areas in which the animals regularly performed non-perambulatory behaviours (e.g. eating, grooming) - the final $10 \mathrm{~cm}$ at either end of the track and $5 \mathrm{~cm}$ around each of the two corners. Similarly, periods when the animals' running speed was less than $3 \mathrm{~cm} / \mathrm{sec}$ were also excluded. Each animal's path was linearized and dwell time and spikes binned into $2 \mathrm{~cm}$ bins, then smoothed with a Gaussian kernel ( $\sigma=5 \mathrm{bins}$ ). Firing rate was calculated for each bin by dividing spike number by dwell time. Separate ratemaps were generated for runs in the outbound and inbound directions.

Replay events from the rest session were identified based on the activity of hippocampal place cells using a similar method to Pfeiffer and Foster ${ }^{7}$ and Olafsdottir et al. ${ }^{8}$ Hippocampal cells were classified as place cells if their firing field's peak firing rate exceeded $1 \mathrm{~Hz}$ and was at least $20 \mathrm{~cm}$ long. Interneurons, identified by narrow waveforms and high firing rates, were excluded from all analyses. To identify replay events, multi-unit (MU) activity from hippocampal place cells only were binned into $1 \mathrm{~ms}$ temporal bins and smoothed with a Guassian kernel $(\sigma=5 \mathrm{~ms})$. Periods when the MU activity exceeded the mean rate by 3 standard deviations were identified as putative replay events. The start and end points of each putative replay event were determined as the time when the MU activity fell back to the mean. Events less than $40 \mathrm{~ms}$ long or which included activity from less than $15 \%$ of the recorded place cell ensemble were rejected (4382 events included in total).

To analyse modulation of grid cell activity by replay events we firstly estimated the firing rate of each cell inside ('event rate') and outside ('baseline rate') events. Difference in event and baseline rates were then assessed using a paired t-test. Secondly, to assess the temporal synchrony between grid cell activity and replay events, we generated peri-stimulus time histograms (PSTH) for each event, centred on the temporal midpoint of the event (bin size $=10 \mathrm{~ms}$ ). Specifically, for each event the number of grid cell spikes emitted in a $2000 \mathrm{~ms}$ window centred on the middle of the event were counted. Spikes were then summed across all recorded events to generate one PSTH per session. Finally, PSTHs for all sessions were combined into a single grand PSTH, the peak of which was identified. The same procedure was repeated for place cells.

To analyse replay, place cell spikes from putative events were binned into $10 \mathrm{~ms}$ temporal bins and a Bayesian framework ${ }^{9}$ was used to calculate the probability of the animal's presence in each spatial bin given the observed spikes - the posterior probability matrix. This approach was validated using data from the track running session except that spikes were binned into $500 \mathrm{~ms}$ temporal bins and location was decoded from the posterior probability matrix using a simple maximum likelihood method. Within each temporal bin the animal's location was decoded to the bin with the highest posterior probability 
and this was compared with the known true location (mean decoding error $20 \mathrm{~cm}$ ). Note, two posterior probability matrices were generated for each event - one for inbound runs and one for outbound runs.

To score the extent to which putative replay events represented a constant speed trajectory along the Z-track we applied a line-fitting algorithm ${ }^{8}$. Lines were defined with a gradient $(V)$ and intercept $(c)$, equivalent to the velocity and starting location of the trajectory. The goodness of fit of a given line $(R(V, c))$ was defined as the proportion of the probability distribution that lay within $30 \mathrm{~cm}$ of it. Specifically where $P$ is the probability matrix:

$$
R(V, c)=\frac{1}{n} \sum_{t=0}^{n-1} P(|x(t)-(V \cdot t . T+c)| \leq d)
$$

where $t$ indexes the time bins of width $T$ and $d$ is set to $30 \mathrm{~cm} . R(V, c)$ was maximised using an exhaustive search to test all combinations of $V$ between $-50 \mathrm{~ms}^{-1}$ and $50 \mathrm{~ms}^{-1}$ in $0.5 \mathrm{~ms}^{-1}$ increments (excluding slow trajectories with speeds $>-2 \mathrm{~ms}^{-1}$ and $<2 \mathrm{~ms}^{-1}$ ) and $c$ between $-15 \mathrm{~m}$ and $21 \mathrm{~m}$ in $0.01 \mathrm{~m}$ increments.

To assess putative replay events for significance we carried out a spatial shuffle of the place cell ratemaps. Specifically, each ratemap was shuffled by shifting it relative to the track by a random number of bins drawn from a flat distribution between 1 and the length of the track minus 1 bin. The ratemap for each cell was rotated independently and in each case trailing bins were wrapped around to ensure an equal number of bins were used for each shuffle. This process was repeated 100 times for each event and for each shuffle we calculated a goodness of fit measure (as described above). This enabled us to estimate the probability of obtaining a given event by chance. Robust events, with an individual $p$ value of less than 0.2 , were accepted as replay events and submitted to further analyses (1826 out of 4382 putative events). Note, two shuffling distributions were generated for each event - one for inbound runs and one for outbound runs. An event was considered to be inbound run if the $p$-value for the inbound run was lower than that for outbound runs, and vice versa. Thus, only one event (either inbound or outbound) was submitted to further analyses. Finally, for all inbound events the grid cell ratemaps for inbound runs were used to assess grid-place cell coherence, and conversely for outbound events grid cell ratemaps for outbound runs were used.

To investigate spatial coherence between grid and place cells during replay events we applied the same Bayesian framework to the grid cell spikes. Hence, for each replay event we also calculated a posterior probability matrix based solely on the observed grid cell spikes. Rather than fitting straight-line trajectories to the periodic grid cell posteriors, we compared the best-fit line from the concurrently recorded place cell posterior. Specifically, we fitted a line with the same intercept and slope as the concurrent place cell event and calculated the proportion of the probability distribution lying within $x / 2 \mathrm{~cm}$ of the line. Where $x$ was equal to the average size of the grid cell firing fields recorded from that animal on the linear track. This value we used to index grid-place cell replay coherence. To estimate statistical significance of the observed coherence scores we used three different shuffling procedures.

In the first instance a shuffle distribution was generated by randomly pairing each grid cell posterior with 100 non-concurrent place cell events from the same animal and from the same session; only place cell 
events that were also accompanied by grid cell firing were used. The line fitting procedure to estimate grid-place cell replay coherence, described above, was re-run. To assess the statistical significance of the obtained distribution of coherence scores against the shuffle we bootstrapped the data distribution 10,000 times, computing the cumulative distribution and the corresponding area-under-the-curve (AUC, i.e. the sum of the cumulative distribution) for each bootstrap. Difference scores between each of the 10,000 AUC scores obtained from the bootstrapped data and the shuffle distribution were computed and the $95 \%$ confidence interval estimated based on these difference scores (assuming a 2-sided test). A result was deemed statistically significant if the confidence interval did not contain 0 .

Second, we applied a spatial shuffling procedure. This procedure was similar to the shuffling procedure used for place cell events. Specifically, each grid cell ratemap was shuffled by shifting it relative to the track by a random number between 10 and the length of the track minus 10 bins. The ratemap for each cell was rotated independently and trailing bins were wrapped around to ensure an equal number of bins were used for each shuffle. This process was repeated 100 times for each event. For each shuffle, the grid-place cell replay coherence score was calculated using the slope and intercept parameters of the best-fit line of the accompanying place cell event (unshuffled). To assess statistical significance we used an AUC test as described above.

Third, we applied a temporal shuffling procedure. Specifically, we shifted the spike times of grid cells active in replay events, by a random amount between $5 \mathrm{~ms}$ and the length of the event minus $5 \mathrm{~ms}$. The relative timing of spikes from the same cell were maintained. Trailing spikes were wrapped around to the start of the event to ensure an equal number of spikes contributed to each shuffle. For each shuffled event, its posterior probability matrix was generated and the best-fit line from the concurrent place cell event (unshuffled) compared to the shuffled posterior and a grid-place cell replay coherence score computed, as detailed above. Statistical significance was again assessed using an AUC test.

Finally, we employed a second, distinct approach, to validate our observation of coherence between grid and place cells during replay. Namely, for each grid cell we constructed an 'event ratemap' by using the timing of grid cell spikes during replay events and the decoded location based on the line fit to the place cell posterior probability matrix. Spikes and dwell time were binned into $2 \mathrm{~cm}$ spatial bins and the resulting ratemap smoothed with a Gaussian kernel ( $\sigma=5$ bins). Each event ratemap was compared with the standard ratemap derived from normal track running using a Pearson correlation. As before, significance was established independently against two null distributions. First, for the 'event ratemap' the correlation between each event and standard ratemap was percentile ranked against correlations obtained between the same ratemaps but with the standard ratemap shifted incrementally to all possible lags relative to the event ratemap - trailing bins were wrapped around to ensure a constant number of bins were used in each comparison. A Wilcoxon signed-rank test was used to determine if the ranks observed for the grid cells differed significantly from an expected median rank of $50 \%$. A second null distribution was generated by shifting the timing of the grid cell spikes within each replay event by a random period drawn from a flat distribution covering 0 s to the length of the replay event. Spikes which exceed the duration of the event were 'wrapped' back to the start but otherwise the relative timing of spikes within each event were unaffected. This procedure was repeated for each event and the event 
ratemap constructed as before and then compared with the standard ratemap. This entire procedure was repeated 100 times for each grid cell and in each case the correlation between the event ratemap and standard ratemap was percentile ranked against the correlations obtained from the shuffling procedure. A Wilcoxon signed-rank test was again used to determine if the observed ranks exceed that expected by chance.

Forward and reverse replay events were identified on the basis of the gradient of the line fit to the place cell posterior probability matrix. For outbound events, events that progress down the track (e.g. from the start of the track to the end) were categorised as forward events, while events depicting runs in the opposite direction were categorised as reverse events. The converse was true for inbound events. To assess whether grid cells were more likely to be active in forward rather than reverse events a chisquare test was used to assess the proportion of all forward events accompanied by grid spikes (57\%) against the proportion of all reverse events accompanied by grid spikes (45\%). Moreover, we repeated the main AUC analysis, described earlier, for each event type to assess difference in grid-place cell coherence for forward and reverse events. Finally, as we recorded more forward events than reverse events (639 vs 315 ) we down-sampled the coherence scores for the forward events a 100 times to equal the number of reverse event coherence scores. For each iteration of the down-sampling procedure we computed the mean forward coherence score and compared it against that for the reverse events. If the mean for 95/100 down-sampling iterations still exceeded that for the reverse events we deemed the obtained statistical difference between forward and reverse coherence scores not to be a confound of varying sample sizes.

To analyse the temporal synchrony between grid and place cell representations during replay events, we applied a time shift to grid cell spikes (starting at $-60 \mathrm{~ms}$ then advancing in $5 \mathrm{~ms}$ steps to $+60 \mathrm{~ms}$ ). For each time shift we calculated the mean coherence between grid and place cells using the line fitting procedure. To assess whether the mean coherence varied as a function of time shift we used a Repeated Measures ANOVA for the forward and reverse events separately. To estimate the peak in this distribution it was mean normalised and fminsearch (Matlab 2015a, Mathworks) used to minimise the RMS difference between the data and a mean normalised Gaussian function in which the centre, height, and standard deviation were allowed to vary. The centre of the fitted distribution was used to define the peak lag.

Finally, we compared the grid-place cell coherence exhibited by the grid cells against their directional modulation and hexagonal symmetry (i.e. gridness). The mean coherence for each grid cell estimated from all events was compared against the $\mathrm{KL}^{5}$ divergence and modified gridness scores ${ }^{3}$ obtained from the foraging sessions using a Spearman rank order correlation. Furthermore, we assessed the statistical significance of grid-place cell replay coherence scores for directional (KL divergence $>0.15$ ) 5.6 and non-directional ( $K L$ divergence $<=0.15$ ) cells separately, using the same AUC test described above. Moreover, we carried out the aforementioned time-shift analysis separately for directional and non-directional cells as well. Finally, we also directly compared the replay coherence of the directional cells with the non-directional cells using a two-sample t-test. 
To assess place cell replay events for statistical significance we used a non-parametric spatial shuffle described above and percentile ranked each event against its own shuffle. Similarly, to assess gridplace cell coherence for significance we used a non-parametric area under the curve (AUC) test, using $95 \%$ confidence intervals to assess statistical significance (if the confidence interval did not contain 0 we deemed a result significant). For the alternative 'event ratemap' method (see above) we used the Wilcoxon signed rank non-parametric test. Spearman rank-order correlation were used to assess relationship between grid-place cell coherence and 'gridness' and directional modulation of grid cells. Finally, to assess bias for grid cell participation in forward (vs reverse) replay events we used a chisquare test. None of the described non-parametric tests have any underlying assumptions (such as normality and equality of variance).

Parametric test were only used to assess difference in grid cell rate during and outside replay events (paired t-test), grid-place cell coherence for directional and non-directional cells (independent samples t-test), and variation in grid-place cell coherence across different grid cell time shifts (repeated measures ANOVA). Data distributions for these tests were assumed to be normal but this was not formally tested.

All tests were two-sided. A supplementary methods checklist is available on request. No statistical methods were used to pre-determine sample sizes but our sample sizes are similar to those reported in previous publications ${ }^{19,20}$.

\section{Histology}

Rats were anaesthetised ( $4 \%$ isoflurane and $4 \mathrm{~L} / \mathrm{min}_{2}$ ), injected intra-peritoneal with an overdose of Euthatal (sodium pentobarbital) after which they were transcardially perfused with saline followed by a $4 \%$ paraformaldehyde solution (PFA). Brains were carefully removed and stored in PFA which was exchanged for a 4\% PFA solution in PBS (phosphate buffered saline) with $20 \%$ sucrose $2-3$ days prior

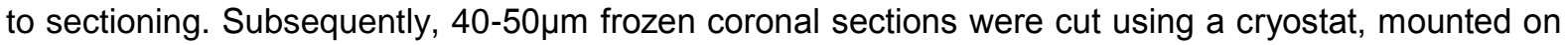
gelatine-coated glass slides and stained with cresyl violet. Images of the sections were acquired using a Leica microscope (DM5500) Sections in which clear tracks from tetrode bundles could be seen were used to determine the location of cells recorded.

\section{Data/code availability}

The data that support the findings of this study are available from the corresponding authors upon request. Analysis software novel to this study can been found in the Supplementary Software file.

14. Barry, C. et al. Nat Neurosci 10, 682-684, doi:10.1038/nn1905 (2007).

15. Wills, T. J., Cacucci, F., Burgess, N. \& O'Keefe, J. Science 328, 1573-1576, doi:10.1126/science.1188224 (2010). 
16. Langston, R. F. et al. Science 328, 1576-1580, doi:10.1126/science.1188210 (2010).

17. Sargolini, F. et al. Science 312, 758-762, doi:10.1126/science.1125572 (2006).

18. Doeller, C. F., Barry, C. \& Burgess, N. Nature 463, 657-661, doi:10.1038/nature08704 (2010).

19. Jeewajee, A. et al. Philos Trans R Soc Lond B Biol Sci 369, 20120532, doi:10.1098/rstb.2012.0532 (2014).

20. Pfeiffer, B. E. \& Foster, D. J. Nature 497, 74-79, doi:10.1038/nature12112 (2013).

21. Olafsdottir, H. F., Barry, C., Saleem, A. B., Hassabis, D. \& Spiers, H. J. eLife 4, e06063, doi:10.7554/eLife.06063 (2015).

22. Davidson, T. J., Kloosterman, F. \& Wilson, M. A. Neuron 63, 497-507, doi:10.1016/j.neuron.2009.07.027 (2009). 